\title{
Exon coconversion biases accompanying intron homing: battle of the nucleases
}

\author{
John E. Mueller, Dorie Smith, and Marlene Belfort ${ }^{1}$ \\ Molecular Genetics Program, Wadsworth Center, New York State Department of Health and School of Public Health, State \\ University of New York at Albany, Albany, New York 12201-2002 USA
}

Intron homing in phage $\mathrm{T} 4$ occurs in the context of recombination-dependent replication, by virtue of intron-encoded endonucleolytic activity. After the $t d$ intron endonuclease I-TevI cleaves the intronless recipient 23 and 25 nucleotides upstream of the intron insertion site, exonucleolytic degradation is required for recombination to proceed. This resection process results in coconversion of exon sequences flanking the intron. In a genetic system designed to study coconversion of flanking markers, we demonstrate that although there is a bidirectional polarity gradient, coconversion can be highly asymmetric. Furthermore, we show that the coconversion of flanking markers favors exon I sequences, upstream of the I-TevI cleavage site. These data are consistent with the asymmetric features of the homing pathways that have been invoked for intron mobility in phage T4. Moreover, these results are in accord with the finding that once the $t d$ homing-site substrate is cleaved, I-TevI remains bound to the downstream cleavage product, protecting against exonucleolytic degradation, and thereby limiting the extent of coconversion into exon II. The results suggest that recombination events are influenced by a competition between the homing endonuclease and exonucleases for sequences downstream of the I-TevI cleavage site, thereby implying a role for the homing endonuclease in the repair process.

[Key Words: Phage T4 td intron; coconversion analysis; exonucleolytic degradation; persistent endonuclease binding; recombination pathways]

Received May 20, 1996; revised version accepted July 22, 1996.

The mobilization of group I introns to cognate intronless alleles is dependent on the activity of site-specific endonucleases encoded within these introns (for review, see Dujon 1989; Lambowitz 1989; Perlman and Butow 1989; Belfort 1990; Mueller et al. 1993; Belfort and Perlman 1995). Double-strand-break (DSB) formation in an intronless gene generates DNA ends that invade homologous exon sequences of an intron-containing allele and prime DNA repair synthesis. These events result in intron inheritance by the recipient allele, a process termed intron homing. Coconversion of flanking exon sequences often accompanies intron homing. Exon coconversion is attributable to exonucleolytic degradation of the cleaved recipient, and possibly also to branch migration during recombination.

In phage $\mathrm{T} 4$, the $t d$ intron-encoded endonuclease, I-TevI, cleaves the recipient allele 23 and 25 nucleotides upstream of the intron insertion site (Bell-Pedersen et al. 1990; Chu et al. 1990). The eccentric cleavage by I-TevI requires both $5^{\prime}-3^{\prime}$ and $3^{\prime}-5^{\prime}$ exonuclease activities to expose exon II sequences for strand invasion and ensure precise intron insertion (Clyman and Belfort 1992). This is consistent with $100 \%$ coconversion of polymorphic

\footnotetext{
${ }^{1}$ Corresponding author.
}

markers between the cleavage site and the intron insertion site, as well as the observed bidirectional gradient of coconversion into distal sequences (Bell-Pedersen et al. 19891.

Functional studies on intron mobility in a phage T4 system indicated that once the recipient allele is cleaved, homing occurs in the context of recombination-dependent DNA replication (George and Kreuzer 1996; Mueller et al. 1996). Specific replication and recombination activities required for intron homing were partially consistent with utilization of the DSB repair (DSBR) pathway to effect intron inheritance. However, the ambiguous requirement for Holliday junction resolvases and the under-representation of crossover recombination products suggested that intron homing occurs via multiple pathways. The synthesis-dependent strand annealing (SDSA) pathway has been invoked as a plausible adjunct to DSBR. Accordingly, Holliday junction intermediates are not formed; therefore, the need for resolvase activity is obviated, and crossover products are not generated (Mueller et al. 1996). Additionally, the extensive chromosome replication (ECR) pathway has been invoked to describe the repair of DSBs in a unimolecular plasmidbased system during phage T4 infection /George and Kreuzer 1996).

Although no direct role for homing endonucleases in the recombination processes has been demonstrated, 
physical studies on the interaction of these endonucleases with their DNA substrates are consistent with their involvement (Plessis et al. 1992; Perrin et al. 1993; Loizos et al. 1996). In this context, it is interesting that I-TevI interacts as a monomer with a 35- to 37-bp region of the $t d$ homing site (Bryk et al. 1995; Mueller et al. 1995). Furthermore, enzymatic and chemical footprinting analyses, defining the interaction of I-TevI with the $t d$ homing site, have shown that DNA sequences flanking the intron insertion site comprise the primary binding site for the endonuclease (Bell-Pedersen et al. 1991; Bryk et al. 1993). Given the asymmetric nature of the I-TevI: $t d$ homing site interaction, it is conceivable that once the DSB is generated, I-TevI remains bound to the product and influences subsequent homing events.

In this paper, we demonstrate that in the phage system, intron homing is associated with asymmetric coconversion of flanking sequences, an observation that can be reconciled with exonucleolytic degradation in the context of the proposed recombination pathways. Furthermore, we show that coconversion of sequences in exon II is limited compared with that in exon I, consistent with the delayed release of I-TevI from the downstream cleavage product and the protective effect of the endonuclease against nucleolytic degradation.

\section{Results}

Intron homing is associated with asymmetric coconversion of flanking markers

To explore the relationship between the frequency and extent of coconversion in the two exons, we exploited the ability of phage T4 to act as a transducing agent (Wilson et al. 1979; Kreuzer and Alberts 1986) and package intron-recipient plasmids following infection by a phage T4 intron donor (Fig. 1; Clyman and Belfort 1992). Restriction site polymorphisms created between donor and recipient were used to examine the coconversion of exon markers. Single homing events from 52 independent infections were examined by restriction analysis, and coconversion was detcrmined by loss of restriction sites in the recombinant product.

The data indicate that the transfer of flanking markers is directly related to the distance separating the marker and the I-TevI cleavage site (Fig. 2A), in agreement with previous studies (Bell-Pedersen et al. 1989). However, there were three unexpected findings. First, the frequency of coconversion of exon I markers was greater than that of exon II markers at cquivalent distances, as reflected by a shift between the two frequency curves (Fig. 2A). Second, at least as many of the homing reactions resulted in grossly asymmetric transfer of the polymorphic sites as in symmetric coconversion (Fig. 2B). Of 22 individual mobility events that could be classified with confidence regarding coconversion bias (Fig. 2, legend), 13 had coconversion tracts on one side of the DSB that were at least five times longer than coconversion tracts on the opposite side. This compared with nine symmetric events displaying less than a twofold bias.

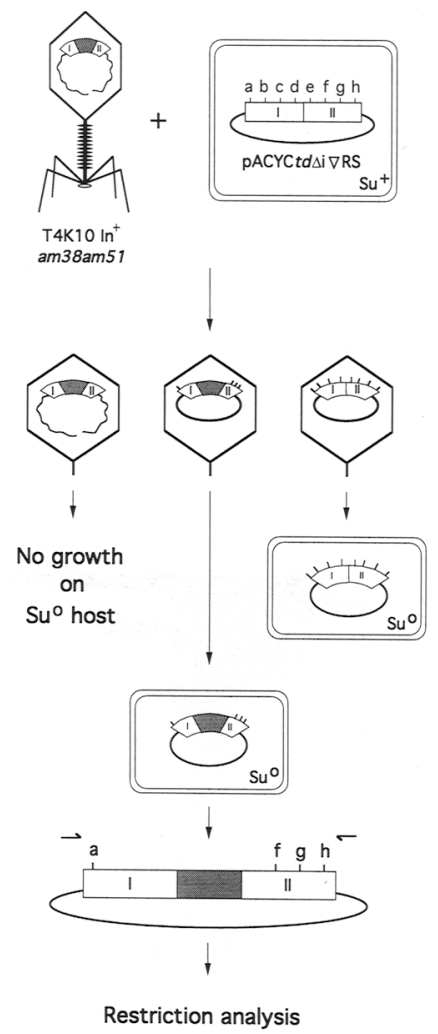

Figure 1. Transduction assay for coconversion of flanking markers during intron homing. The intron (shaded box) moves from a $\mathrm{T} 4 \mathrm{~K} 10$ donor to a $\mathrm{Tet}^{\mathrm{R}}$ recipient plasmid pACYCtd $\triangle \mathrm{i} \nabla \mathrm{RS}$, which contains eight unique restriction sites $(a-h)$ not present in donor exons. Intron-containing Tet ${ }^{R}$ transductants arising from delivery of plasmid DNA by T4K10 into a Su" host were subjected to PCR-based restriction analysis with each of the eight unique restriction enzymes. Restriction sites a-h correspond to: (a) NaeI; (b) BssHII; (c) NarI; (d) SpeI; (e) BamHI; (f) ApaI; (g) XmnI; (h) MluI. Single transductants from independent infections were assayed.

Third, among the asymmetric events, coconversion into exon I ( 10 of 13 events) dominated over that into exon II ( 3 of 13 events) (Fig. 2B), in agreement with the general tendency of marker coconversion favoring exon I (Fig. 2A).

Homing endonuclease I-TevI remains bound to downstream cleavage product

The limited coconversion of exon II sequences (Fig. 2) suggested that I-TevI remains bound to its downstream cleavage product and protects exon II from nucleolytic degradation. This idea was reinforced by the fact that I-TevI makes primary contacts with its DNA substrate at sequences flanking the intron insertion site, downstream of the I-TevI cleavage site (Fig. 3A) (Bell-Pedersen et al. 1991; Bryk et al. 1993). To test the hypothesis that I-TevI remains bound to the DNA after cleavage, we performed electrophoretic analyses to examine the interaction of the endonuclease with upstream and downstream 
A

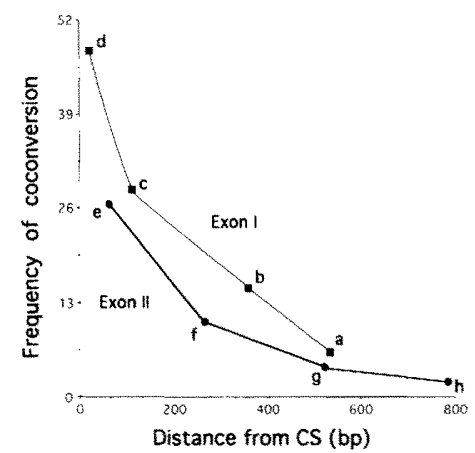

B

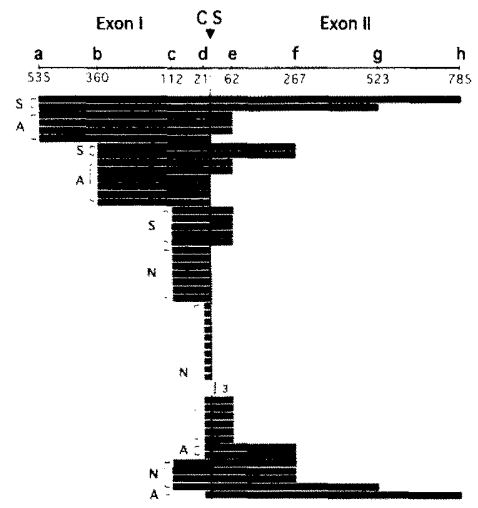

Figure 2. Coconversion analysis. (A) Quantitative analyses of combined coconversion events. The frequency of coconversion, determined by the loss of a restriction site in the recombinant product, was plotted as a function of the distance (base pairs) between the I-TevI cleavage site (CS) and the restriction site. Exon $I_{i}(-)$ exon II. Restriction sites a-h correspond to those in Fig $L$ and $2 B$ and are listed in the legend to Fig 1. $(B)$ Quantitative analyses of individual coconversion events. Map of eight poly* morphic restriction endonuclease sites indicates distances from I-TevI cleavage site. Coconversion tracts (black bars) for 52 independent transductants were plotted. Three recombinants ex hibited no coconversion of markers. When progeny of individual transductants were examined by restriction analysis, all exhibited identical coconversion profiles to the parental transductant indicating that the parental transductant represented a single coconversion event. Symmetric events, where coconversion on the two sides varied by less than twofold, are marked S (nine totall, whereas asymmetric events, where coconversion on the two sides varied by greater than fivefold are marked A | 13 totall. The other 30 events $(N)$ were not classified because values fell between these ranges or because coconversion tracts were too short.

cleavage products. Upon incubation with its DNA substrate under $\mathrm{Mg}^{2+}$-plus cleavage conditions, I-TevI interaction with the upstream cleavage product was not detectable after cleavage was complete (Fig. 3B, lanes 1,2). In contrast, I-TevI remained bound to the downstream cleavage product (Fig. 3B, lanes 5,6 ) that contains the primary binding site for the endonuclease (Fig. 3A). Gel mobility-shift analyses were also performed with purified cleavage products. Again, the results indicate that the downstream cleavage product contains the necessary recognition signals to bind the endonuclease, whereas the upstream cleavage product does not (Fig. 3B, cf. lanes 7 and 8 with lanes 3 and 4). Thus, not only does I-TevI remain associated with one of its products, but it has the potential to bind the downstream product independently.

To further examine interactions with I-TevI, incubation was performed in the absence of $\mathrm{Mg}^{2+}$ under conditions in which the enzyme can bind, but not cleave, its substrate. Under such conditions, the full-length substrate forms two complexes with I-TevI in a gel mobilityshift assay (Fig. 3C, lane 2, $\mathrm{U}_{\mathrm{S}}$ and $\mathrm{U}_{\mathrm{F}}$ ), as observed previously by Bryk et al. (1995) and Mueller et al. (1995), whereas the downstream cleavage product forms a single complex (Fig. 3C, lane 4). When limiting quantities of
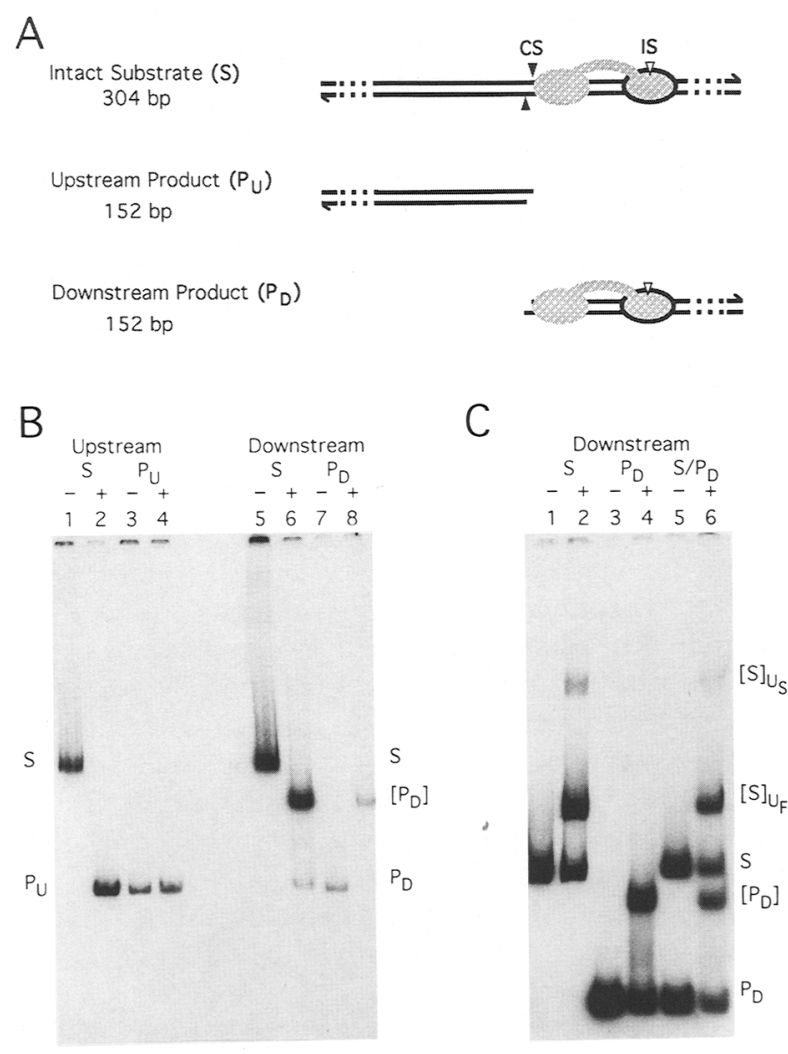

Figure 3. I-TevI binding to its cleavage product. (A) Schematic representation of DNA substrate and cleavage products. (Solid arrowheads and CS) I-Tevl cleavage site; lopen arrowhead and IS) intron insertion site. Shading represents I-TevI, with the outlined region defining interaction of the DNA-binding domain of I-TevI with the primary binding site on the DNA (adapted from Bryk et al. 1995; Mueller et al. 1995). (S) intact 304-bp substrate. $\mathrm{P}_{\mathrm{U}}$ and $\mathrm{P}_{\mathrm{D}}, 152$-bp upstream- and downstream-cleavage products, respectively. $(B)$ Electrophoretic analysis of I-TevI:td homing site interaction in the presence of $\mathrm{Mg}^{2+}$. DNA fragments $S$, $P_{U}$ or $P_{D}$ were incubated in the absence $(-)$ or presence $(+)$ of I-TevI and separated on $8 \%$ polyacrylamide gels. (Upstream and Downstream) Labeled $5^{\prime}$ ends of DNA substrate. (Brackets) I-TevI-shifted DNA. Other labels as in A. (C) Mobilityshift analysis of intact substrate and downstream cleavage product in the absence of $\mathrm{Mg}^{2+}, \mathrm{U}_{\mathrm{S}}$ and $\mathrm{U}_{\mathrm{F}}$, catalytically active I-TevI:DNA complexes (Bryk et al. 1995). 
I-TevI were incubated with equimolar amounts of an intact $t d$ homing-site fragment and its respective downstream product, the relative amount of binding by the endonuclease for each species was comparable to that amount when only a single substrate was used in the analysis. Thus, $58 \%$ of the DNA was bound by I-TevI, when the intact $t d$ homing site served as substrate (Fig. $3 \mathrm{C}$, lane 2 ); and in the mixing experiment, $55 \%$ of the intact homing site was bound by the endonuclease /Fig. $3 \mathrm{C}$, lane 6). Likewise, when the cleavage product was the sole substrate in the analysis, $49 \%$ of the downstream product was bound by I-TevI (Fig. 3C, lane 4); and in the mixing experiment, $50 \%$ of the downstream product was bound (Fig. 3C, lane 6). These data indicate that I-TevI has comparable binding affinities for the intact $t d$ homing site and the downstream cleavage product and are in accord with the ensuing intron-mobility event bcing influenced by the delayed release of I-TevI from the downstream cleavage product.

\section{The effect of I-TevI binding on exonucleolytic degradation}

To examine the effect of the delayed release of I-TevI from downstream sequences on nucleolytic activity, degradation analyses were performed on recipient plasmid that had been incubated with I-TevI and T4-infected cell extracts. Experiments were designed such that degradation into sequences upstream and downstream of the I-TevI cleavage site were monitored from the same cleaved substrate and to the same distance into each exon on both strands, as defined by the primer binding sites (see Materials and methods and schematic representations in Figs. 4 and 5). To examine $5^{\prime}-3^{\prime}$ degradation, quantitative primer-extension analyses were performed by use of primers $d$ and $i$ specific to exon I and exon II sequences, respectively (Fig. 4A). Each primer generated the expected 152-nucleotide run-off fragment from the I-TevI-cleaved recipient plasmid (Fig. 4B, inset, $0 \mathrm{~min} /$. When the plasmid was incubated with I-TevI (derivative $\mathrm{H} 40 \mathrm{Y}$, see Materials and Methods) and T4infected cell extracts prior to analysis, nuclease activity was manifest by a reduction in intensity of the 152-nucleotide band (Fig. 4B, 5-30 min). Consistent with the protective effect of I-TevI, degradation into exon II sequences was significantly reduced compared with that into exon I over $30 \mathrm{~min}$ incubation (Fig. 4B). When I-TevI was not included in the incubation, degradation of the I-TevI-cleaved substrate was equivalent in both exons (data not shown).

To corroborate these results and to facilitate eventual examination of $3^{\prime}-5^{\prime}$ degradation, hybridization analysis was performed by use of primers $\mathrm{d}$ and $\mathrm{i}$ as probes /Fig. $4 \mathrm{Cl}$. In a time-course analysis over $20 \mathrm{~min}$, degradation into exon I was again more rapid and affected a larger fraction of molecules than degradation into exon II.

Next, both $5^{\prime}-3^{\prime}$ and $3^{\prime}-5^{\prime}$ exonucleolytic degradation were examined at precise and equivalent distances into the two exons, as defined by multiple primer binding sites (Fig. 5A). Representative data from a nuclease ac-
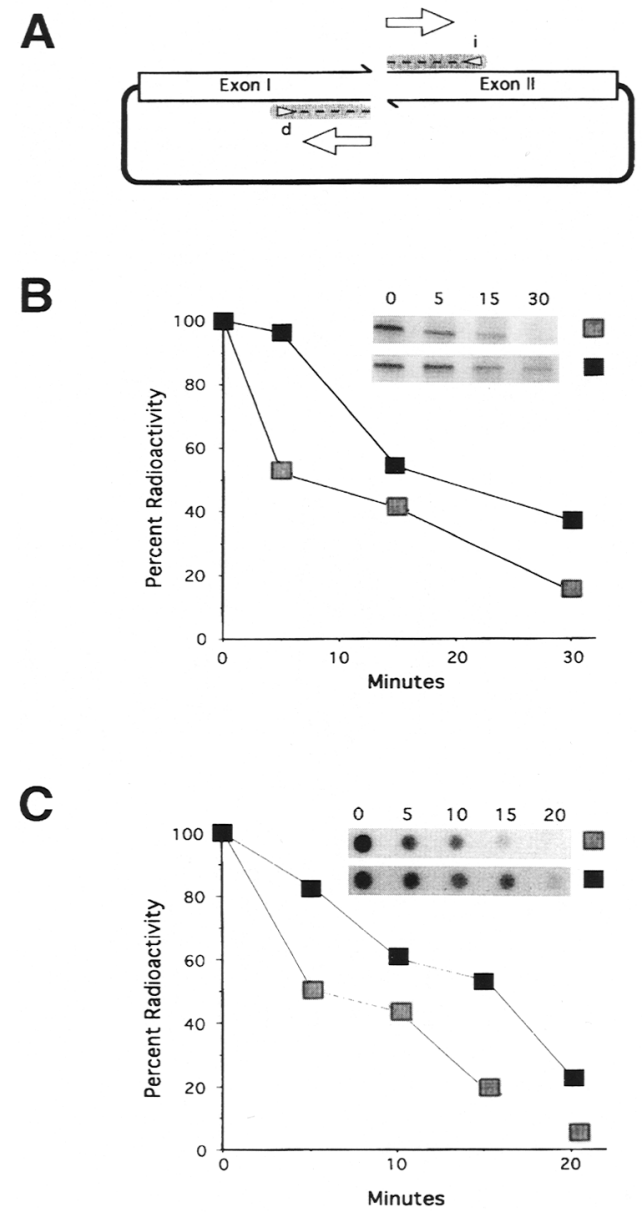

Figure 4. Timecourse analysis of $5^{\prime}-3^{\prime}$ degradation into exon scquences. (A) Schematic of assays. Primers d and i larrowheads), centered around $140 \mathrm{bp}$ from the cleavage site, were used to examine $5^{\prime}-3^{\prime}$ degradation (open arrows) into exon I and exon II, respectively. Primer extension analysis monitors disappearance of a 152-nucleotide extension product (shaded) for each exon (panel B), whereas hybridization analysis monitors diminution of a hybridization signal (panel C). (B) Primer-extension analysis. Primer-extension products (inset with incubation times) were quantitfied and the percent radioactivity relative to the 0 min incubation plotted. (Shaded boxes) Exon 1; (solid boxes) exon 2. (C) Hybridization analysis. Dot blots (inset with incubation times) were quantified and relative intensities plotted as a function of incubation time in phage T4-infected cell extracts. Other labels as in Fig. 4B.

tivity analysis at the $20 \mathrm{~min}$ timepoint are displayed graphically in Figure $5 \mathrm{~B}$. Both $5^{\prime}-3^{\prime}$ and $3^{\prime}-5^{\prime}$ degradation of exon I were more extensive than that of exon II, and furthermore, this degradation bias exhibited distance-dependence, being more clearly apparent proximal to the cleavage site than at distal sequences (Fig. 5B cf. exon I and exon II at distances of 10 to 140 nucleotides versus 270 nucleotides). These data are in accord with the in vivo coconversion analysis in which the disparity in frequencies between the two exons was also more dramatic for markers proximal to the cleavage site than 
A

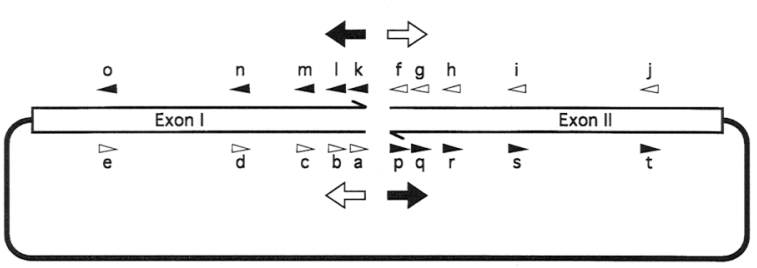

B

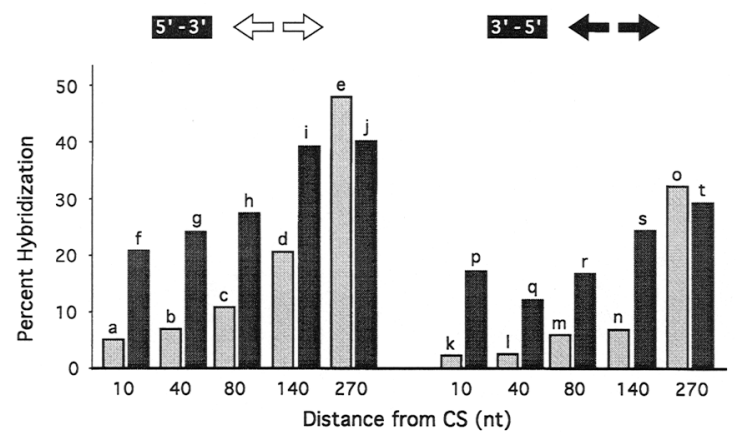

Figure 5. Distance-dependence of $5^{\prime}-3^{\prime}$ and $3^{\prime}-5^{\prime}$ exonucleolytic degradation into exon sequences. (A) Schematic of hybridization probes. Primers a-e and $f-i$ (open arrowheads) were used to measure $5^{\prime}-3^{\prime}$ degradation (open arrows) into exon I and exon II, respectively. Primers $\mathrm{k}-\mathrm{O}$ and $\mathrm{p}-\mathrm{t}$ (solid arrowheads) were used to examine 3 ' -5 ' degradation (solid arrows) into exon I and exon II, respectively. For precise primer coordinates, see Materials and Methods. $(B)$ Distance-dependence of nucleolytic degradation. Shaded bar, exon I; solid bar, exon II. Intensities of quantified dot blots for 20 -min incubations relative to 0 -min incubations were plotted as a function of approximate distance (nt) of probe from I-TevI cleavage site. Analyses were performed at least two times for each distance with degradation profiles exhibiting similar trends. Probes for each hybridization are indicated above bars.

for distal markers (Fig. 2A). Together, the results indicate that the interaction of I-TevI with the downstream cleavage product protects exon II sequences from both $5^{\prime}-3^{\prime}$ and $3^{\prime}-5^{\prime}$ exonucleolytic degradation, in accord with the genetic data demonstrating limited coconversion into this exon.

\section{Discussion}

Coconversion biases were manifest in several different ways when exon marker coinheritance with the intron was measured in our transduction assay. First, on average, coconversion of markers occurred with greater frequency in exon I than in exon II (Fig. 2A). Second, for independent events, highly asymmetric coconversion tracts were as frequent as symmetric events in the two exons (Fig. 2B). Third, the lengthy asymmetric events into exon I outnumbered those into exon II by about $3: 1$ (Fig. 2B). The disparity in coconversion frequency in the two exons, with more limited coconversion into exon II, was viewed in the context of I-TevI remaining bound to its downstream cleavage product (Fig. 3). The data indi- cate that sequences downstream of the I-TevI cleavage site, which include the primary binding site for the endonuclease (Bell-Pedersen et al. 1991; Bryk et al. 1993), are initially guarded from nucleolytic processing into exon II by I-TevI binding (Figs. 4 and 5). Under such circumstances, exonucleolytic degradation of downstream sequences, which is required for homing and coconversion into exon II, is presumed to reflect competition for binding between the mobility endonuclease and degradative exonuclease(s) (see below).

In accord with the observed exon degradation biases are experiments in which there is heterology between intron donor and recipient, where there is a demand for extensive resection of the cleaved recipient before homologous sequences are reached (Parker et al. 1996). In such cases, degradation of exon I sequences upstream of the I-TevI cleavage site was favored over degradation of downstream sequences. Thus, homing frequencies were $25 \%$ higher when sequence heterology between donor and recipient existed on the exon I side, than on the exon II side of the I-TevI cleavage site. The protective effect of persistent $\mathrm{I}-\mathrm{TevI}$ binding is thus manifest in vivo under very different genetic circumstances.

The combination of symmetric and asymmetric marker coconversion events may be viewed in terms of multiple pathways being utilized for intron homing (Mueller et al. 1996; George and Kreuzer 1996). Although the intermolecular nature of our plasmid-based assay does not allow us to evaluate our results in the context of the ECR pathway, asymmetric coconversion of flanking markers over single events could be envisaged for either the DSBR pathway or SDSA pathway for intron mobility. An underlying assumption in the following arguments is that association with donor sequences protects the recipient ends from nucleolytic degradation (White and Haber 1990; Sweetser et al. 1994). The exon sequences involved in strand invasion would thereby be afforded protection at the onset of repair synthesis, whereas the noninvading exon would be subject to exonucleolytic degradation until it became associated with complementary template sequences for repair synthesis. It could be argued, however, that the asymmetry would be exaggerated for the SDSA pathway because of the way in which the noninvading strand initiates repair synthesis (Fig. 6). A major difference between the two pathways lies in the origin of the template for repair synthesis of the noninvading strand. For DSBR, the donor allele serves as template for repair synthesis (Fig. 6A, stage 5), whereas for SDSA it is the recipient allele (Fig. 6A, stage $6^{\prime}$ ). Thus, during DSBR, repair synthesis of the noninvading strand of the recipient depends on sequences at the leading edge of the displaced D-loop of the donor (Fig. 6A,B, stage 5). In contrast, for SDSA, it is the newly synthesized strand, released from the trailing edge of the replication bubble, that serves as template for repair synthesis of the noninvading strand (Fig. 6A, stage 6'). Therefore, during SDSA, the size of the replication bubble influences the availability of repair template (Fig. 6B) and, consequently, the extent of degradation of the exposed, noninvading strand. 
A

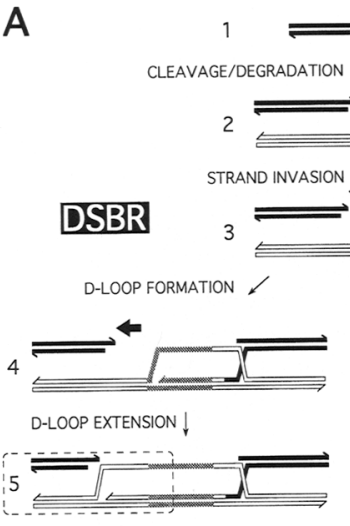

REPAIR SYNTHESIS $\downarrow$

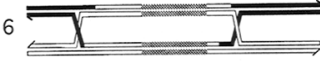

RESOLUTION $\downarrow$

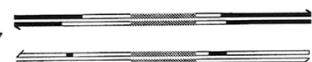

SDSA

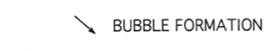

$4^{\prime}$

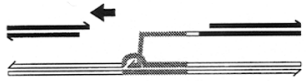

| BUBBLE MIGRATION

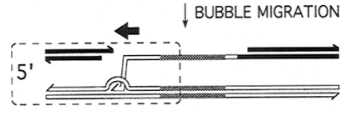

$\downarrow$ ANNEALING

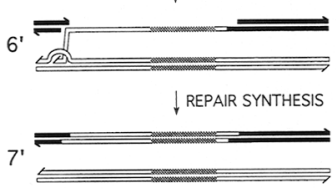

B

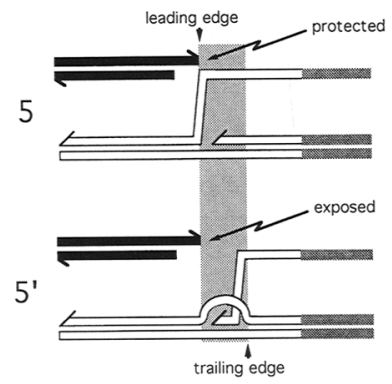

Figure 6. DSBR and SDSA pathways and asymmetric processing. (A) Model pathways for intron homing in phage T4 (adapted from Mucller et al. 1996). After clcavage, I-TevI remains bound to the downstream product at the primary binding site (shaded dumbbell) (stage 1). After nucleolytic processing (stages 1-3), strand invasion of an intron-containing allele occurs (stage 3). Solid arrows indicate degradation; corresponding shaded arrow signifies inhibition of degradation by I-TevI. Repair synthesis is initiated and continues through the intron /stages 4-6, DSBR, $4^{\prime}-7^{\prime}$, SDSA). For DSBR, D-loop formation results in the displaced donor strand serving as template for repair synthesis of the non-invading recipient strand (stage 5). Holliday junction intermediates are formed (stage 6), which are resolved to generate homing products. Only the noncrossover product is shown (stage 7). In SDSA, the newly synthesized strand is released from the donor as repair synthesis proceeds (stages $4^{\prime}-6^{\prime}$ ) and serves as template for repair of the noninvading strand (stages $6^{\prime}-7^{\prime}$ ). Half-arrows, 3' end of DNA strand. Shaded strands, intron. D (open strands), donor; R (black strands), recipient. Dashed boxes in $A$ demarcate segments depicted in $B$, with stage 5 representing DSBR and $5^{\prime}$ representing SDSA. (B) SDSA favors exonucleolytic degradation. Shading indicates differences in the availability of template to the noninvading strand, with prolonged exposure to exonucleases in the SDSA pathway.

In the absence of extensive exonucleolytic degradation, the formation of heteroduplex DNA resulting from strand-invasion and branch-migration events could account for the observed coconversion of polymorphic markers. Interestingly, in vivo and in vitro studies have demonstrated that repair tracts in a phage T4 system are locally confined to the mismatch and neighboring sequences (Kleff and Kemper 1988; Shcherbakov and Plugina 1991; Solaro et al. 1993). Therefore, if heteroduplex DNA resulting from branch migration is repaired during infection, one would predict discontinuities in coconversion tracts. We have observed no such discontinuities in our analyses, suggesting that exonucleolytic degradation is the major contributor to coconversion (Fig. 2B; data not shown).

Considcring that I- $\mathrm{TevI}$ binds the downstream cleavage product (Fig. 3B,C) and appears to protect exon II sequences from degradation (Figs. 4 and 5), one must address the fact that resection of sequences between the I-TevI clcavage site and intron insertion site is necessary to ensure precise homing. The way in which I-TevI contacts its substrate suggests a means for exonucleases to access the downstream cleavage product even in the presence of the endonuclease (Fig. 6A; stage 1) (Bryk et al. 1993, 1995; Mueller et al. 1995|. Although I-TevI interacts with two distinct regions of the intronless $t d$ gene, stretching from the intron insertion site to the cleavage site (Fig. 3A), primary contacts made by the endonuclease are limited to 6 nucleotides upstream of the intron insertion site (Bryk et al. 1993). Therefore, nucleolytic degradation from the cleavage site toward the insertion site could begin even in the presence of bound I-TevI. While I-TevI is likely to stall the resection enzyme(s), degradation into exon II would ensue once I-TevI became displaced from its binding site. In light of these observations, the conundrum that exon II sequences need to be exposed for precise homing to occur, yet I-TevI binding protects these sequences from degradation, can be explained by a competition between I-TevI and the degradative exonucleases for the downstream cleavage product.

From the foregoing it would appear that asymmetric coconversion of exon markers during $\mathrm{T} 4$ homing is largely attributable to nucleolytic degradation, which might be influenced by both features of the repair pathway and the persistent binding of I-TevI. Protection from degradation might be achieved by early strand invasion on the one hand, and/or by I-TevI binding on the other. Given the observed asymmetry of coconversion, which is biased toward exon I sequences, one is tempted to ask whether I-TevI merely plays a role in impeding nucleolytic degradation of exon II sequences or whether the endonuclease is further involved in homing. Recombination might be initiated by the endonuclease delivering the downstream sequences to the intron donor thereby stimulating strand invasion, or by recruiting proteins that potentiate homing.

\section{Materials and methods}

DNA oligonucleotides

Primers used for coconversion analyses are as follows: W340, 5'-GTGTAATTGGCGGGCCTGCTCTGTTATATGC-3' 
and W341, 5'-CGCAGCAGCCTTAATGACAATAGTCTG-3'. Probes and primers used for nuclease assays (Fig. 4 and 5) are as follows, with the distance between the center of each primer $(a-t)$ and the I-TevI cleavage site indicated in parenthesis: $\mid \mathrm{a})=$ W605, 5'-TGGATTTGCAGTGGTATCAAC-3' (1) nucleotides); (b) = W609, 5'-TCTATCAGTTTAATGTGCGTA-3' $\mid 41$ nucleotides $) ;(\mathrm{c})=$ W562, 5'-CCAGCTGAACTTAAATATATGGC-3' (85 nucleotides); (d) = W311, 5'-TATTGATCGTATTAAAAAACTGCC-3' (139 nucleotides); (e) =W554, 5' -GGCAAAACAGTCTGGGATG-3' $(270$ nucleotides $) ;(\mathrm{f})=$ W608, 5 $^{\prime}$ AAGAAAACATCTACTGAGCGT-3' (11 nucleotides); $(\mathrm{g})=$ W612, 5'-GCATATGACGCAATATTAAAC- $3^{\prime}$ (41 nucleotides); $(\mathrm{h})=$ W565, 5'-CCCCTGGAATAAGATTACACATCTTAGC3' (83 nucleotides); (i) =W312, 5'-ACATTGTTCTACGTGAT TC-3' (143 nucleotides); (j) = W556, 5'-AACGAAATCTTTAGGCC-3' (276 nucleotides); $(k)=$ W606, 5' ${ }^{\prime}$ TGATACCACTGCAAATCCAAA-3' $(11$ nucleotides $) ;(1)=$ W610, 5' -CGCACAT TAAACTGATAGAAC-3' $(41$ nucleotides $) ;(\mathrm{m})=$ W563, $5^{\prime}$ GCCATATATTTAAGTAGTTCAGCTGG-3' (85 nucleotides); $(\mathrm{n})=$ W553, 5'-GGCAGTTTTTTAATACGATC-3' |137 nucleotides); (o) $=$ W566, 5'-CATCCCAGACTGTTTTGCC-3' $(270$ nucleotides $) ;(\mathrm{p})=\mathrm{W} 607,5^{\prime}$-GCTCAGTAGATGTTTTCTTGG3' (11 nucleotides); $(q)=$ W611, 5'-TTAATATTGCGTCATATGCTA-3' (41 nucleotides); $(\mathbf{r})=$ W564, 5'-GCTAAGATGTGTAATCTTATTCCAGGGG-3' (83 nucleotides); $(\mathrm{s})=\mathrm{W} 555,5^{\prime}$ GAATCACGTAGAACAATGT-3'(143 nucleotides $) ;(\mathrm{t})=\mathrm{W} 567$; 5'-GGCCTAAAGATTTCGTT-3' (276 nucleotides).

\section{Coconversion assay}

Plasmid transduction was performed as described previously (Clyman and Belfort 1992). $\mathrm{Su}^{+}$cells harboring intron recipient plasmid pACYC184td $\triangle \mathrm{i} \nabla \mathrm{RS}\left(\mathrm{Tet}^{\mathrm{R}}\right)$ were infected with $\mathrm{T} 4 \mathrm{~K} 10$ containing amber mutations in genes 38 and 51 (Selick et al. 1988). Progeny phage were then infected into Su" host Escherichia coli $\mathrm{B}$ and the cells grown on tetracycline-containing plates. Single isolated $\mathrm{Tet}^{\mathrm{R}}$ transductants from 52 independent infections were subjected to PCR by use of primers W340 and W341 that are complementary to sequences at the distal regions of homology between donor and recipient. Coconversion of polymorphic restriction sites was determined by restriction analyses with the eight enzymes indicated in the legend to Figure 1 .

\section{Electrophoretic analysis}

Electrophoretic assays were performed with a 304-bp DNA fragment containing the $t d$ homing site (probe n, Mueller et al. 1995). The substrate was either $5^{\prime}$-end labeled on the top strand or the bottom strand, allowing independent monitoring of the upstream or downstream cleavage products, respectively. Upstream and downstream cleavage products used in electrophoretic assays were generated in cleavage reactions containing the labeled 304-bp fragment and homing endonuclease I-TevI (BellPedersen et al. 1990). Accordingly, the specific activity for each labeled intact homing-site fragment and its respective cleavage product were equivalent. All DNA fragments were purified from polyacrylamide gels prior to electrophoretic analysis. $\mathrm{I}-\mathrm{Tevl}$ was synthesized in an in vitro wheat germ extract translation system (Promega, Madison, WI) (Bell-Pedersen et al. 1990).

I-TevI was incubated with DNA fragments in either JBB buffer containing $50 \mathrm{mM}$ Tris- $\mathrm{HCl}$ at $\mathrm{pH} 8.0,20 \mu \mathrm{g} / \mathrm{ml}$ poly[d(I-C), 10 $\mu \mathrm{g} / \mathrm{ml}$ BSA or in MBB buffer containing $25 \mathrm{~mm}$ Tris- $\mathrm{HCl}$ at $\mathrm{pH}$ $8.0,50 \mathrm{~mm} \mathrm{NaCl}, 20 \mathrm{~mm}$ EDTA, $5 \%$ glycerol, $100 \mu \mathrm{g} / \mathrm{ml}$ poly[d(I-C)], $2.5 \mu \mathrm{g} / \mathrm{ml} \mathrm{BSA}$. Considering that JBB contains no metal-chelating agents and wheat germ extracts contain $2.1 \mathrm{~mm}$ magnesium acetate, incubation in IBB resulted in cleavage of the intact substrate by the endonuclease (Fig. 3B). Conversely, in gel mobility-shift analyses with MBB buffer, intact substrate cleavage by I-TevI was not detected because of the presence of $20 \mathrm{mM}$ EDTA (Fig. 3C). In the mixing experiments of Figure 3C, the molar quantities of total DNA fragments in each reaction mixture were equivalent. To ensure that binding experiments were performed under conditions in which I-TevI was saturated with substrate, a series of gel mobility-shift assays, as depicted in Figure 3C, was performed with increasing quantities of I-TevI (data not shown). In all binding analyses, unbound species were incubated with unprogrammed wheat-germ extracts. Polyacrylamide gel electrophoresis was conducted as described previously (Mueller et al. 1995). Gels were quantitated on a Betascope 603 Blot Analyzer as recommended by the manufacturer.

\section{T4-infected cell extracts}

T4-infected cell extracts were prepared according to Morris et al. (1979), with minor modifications. E. coli B cells were grown to a density of $3 \times 10^{8}$ cells per $\mathrm{ml}$ and infected with T4K10 phage (Selick et al. 1988) at an moi of 6.0. Infection proceeded for $17 \mathrm{~min}$, cells were collected by centrifugation and stored at $-80^{\circ} \mathrm{C}$. Cell pellets were resuspended in 0.01 of the original culture volume in $50 \mathrm{~mm}$ Tris- $\mathrm{HCl}$ at $\mathrm{pH} 8.0,25 \%$ sucrose and incubated with $0.2 \mathrm{mg} / \mathrm{ml}$ lysozyme for $45 \mathrm{~min}$ at $4^{\circ} \mathrm{C}$. Extracts were brought to a final concentration of $5 \%$ Triton X-100 (Sigma, St. Louis, $\mathrm{MO}$ ) and incubated for $60 \mathrm{~min}$ at $4^{\circ} \mathrm{C}$. Debris was removed by centrifugation at $30,000 \mathrm{~g}$ for $60 \mathrm{~min}$ at $4^{\circ} \mathrm{C}$. The supernatant was brought to a final concentration of $2.5 \%$ streptomycin sulfate (Sigma, St. Louis, MO), stirred gently for $60 \mathrm{~min}$ at $0^{\circ} \mathrm{C}-4^{\circ} \mathrm{C}$ and nucleic acids were removed by centrifugation at $30,000 \mathrm{~g}$ for $30 \mathrm{~min}$ at $4^{\circ} \mathrm{C}$. Ammonium sulfate /GIBCO-BRL, Gaithersburg, $\mathrm{MD}$ / was added slowly to the supernatant to a final concentration of $350 \mathrm{mg} / \mathrm{ml}$ and the solution stirred gently for $60 \mathrm{~min}$ at $0^{\circ} \mathrm{C}-4^{\circ} \mathrm{C}$. Proteins were precipitated by centrifugation at $30,000 \mathrm{~g}$ for $30 \mathrm{~min}$ at $4^{\circ} \mathrm{C}$, resuspended in $25 \mathrm{~mm}$ Tris- $\mathrm{HCl}$ at $\mathrm{pH} 8.0,1.2 \mathrm{~mm}$ EDTA, $1 \mathrm{~mm} \beta$-mercaptoethanol, $100 \mathrm{~mm} \mathrm{NaCl}$ and dialyzed against the same buffer at $4^{\circ} \mathrm{C}$. Extracts were quick-frozen in liquid nitrogen as $0.1 \mathrm{ml}$ aliquots and stored at $-80^{\circ} \mathrm{C}$.

\section{Nuclease activity assays}

I-TevI-cleaved pBStdd In substrate (250 ng) was incubated with 5 units of purified I-TevI (H40Y) and $5 \mu \mathrm{l}$ of extract from T4infected cells for $5-30 \mathrm{~min}$ at $37^{\circ} \mathrm{C}$ in $50 \mathrm{~mm}$ Tris- $\mathrm{HCl}$ at $\mathrm{pH} 8.0$, $10 \mathrm{mM} \mathrm{MgCl}_{2}$. The I-TevI (H40Y) derivative binds and cleaves the $t d$ homing site with wild-type fidelity, yet exhibits reduced catalytic activity (M. Bryk, D. Smith, and J.E. Mueller, unpubl.; V. Derbyshire, J.C. Kowalski, J.T. Dansereau, C.R. Hauer, and M. Belfort, in prep.). Thus, the enzyme is easily overexpressed and purified allowing stoichiometric amounts of I-TevI to be used in the nuclease assay. Reactions were stopped by phenol extraction and the DNA precipitated with ethanol. Quantitative primer-extension assays were performed by modification of the procedures of Singer-Sam and Riggs (1993) to monitor 5'-3' degradation. Samples were subjected to primer extension with Sequenase 1.0 (USB, Amersham Life Sciences, Cleveland, Ohio) in the presence of $\left[\alpha_{-}{ }^{35} \mathrm{~S}\right] \mathrm{dATP}$, as suggested by the manufacturer, without the addition of ddNTPs. Half of the primer-extension aliquot was analyzed with primer $d$ to examine degradation upstream of the I-TevI cleavage site, while the other half was analyzed with primer i to examine downstream sequences. Primer-extension products were analyzed on $6 \%$ denaturing polyacrylamide gels (Mueller et al. 1995) with a Molecular Dy- 
namics Inc. PhosphorImager using ImageQuant software. Sequencing ladders (not shown) were run alongside the cleavage products to verify the position of the cleavage site and the length of the primer-extension product $(152$ nucleotide for primers $\mathrm{d}$ and $\mathrm{i}$.

For dot-blot hybridization analyses, I-TevI-cleaved pBStdai substrate was incubated with 5 units of $\mathrm{I}-\mathrm{TeVI}(\mathrm{H} 4 \mathrm{OY})$ and extracts from T4-infected cell for $5-20 \mathrm{~min}$ at $37^{\circ} \mathrm{C}$ in JBB buffer, supplemented with $2 \mathrm{mM} \mathrm{MgCl}_{2}$. Reactions werc stopped by phenol extraction and the DNA precipitated with ethanol. Samples were incubated in $1.2 \mathrm{M} \mathrm{NaCl}, 0.4 \mathrm{M} \mathrm{NaOH}$ for $30 \mathrm{~min}$ at $37^{\circ} \mathrm{C}$, neutralized with the addition of 8 volumes of $0.5 \mathrm{M}$ Tris$\mathrm{HCl}$ at $\mathrm{pH} 7.5,1.5 \mathrm{M} \mathrm{NaCl}, 1 \mathrm{~mm}$ EDTA and blotted to Hybond-N (Amersham Corp., Arlington Heights, IL) with a BRL hybri-dot blot system (GIBCO-BRL, Gaithersburg, MD). Hybridization was performed according to Sambrook et al. (1989). Each set of probes was designed such that assays measured 5 ' -3 ' and $3 '-5$ ' degradation to the same region, which corresponds to the binding sites of these complementary primers. I-TevI-cleaved substrate and I-TevI (H40Y) were prepared according to BellPedersen et al. (1989 and 1991, respectively). Dot-blot assays were quantitated on a Betascope 603 Blot Analyzer.

\section{Acknowledgments}

We acknowledge Deb Court and Joe Kowalski for generating the polymorphic substrates used in the coconversion analysis. We thank past and present members of the laboratory for insightful discussions and Mary Bryk, Vicky Derbyshire, Joyce Huang, and Monica Parker for critical comments on the manuscript. We are especially grateful to Maryellen Carl and Maureen Belisle for preparation of the manuscript and illustrations. This work has been supported by grants GM39422 and GM44844 to M.B. and GM15454 to J.E.M. from the National Institutes of Health.

The publication costs of this article were defrayed in part by payment of page charges. This article must therefore be hereby marked "advertisement" in accordance with 18 USC section 1734 solely to indicate this fact.

\section{References}

Belfort, M. 1990. Phage T4 introns: Sclf-splicing and mobility. Ann. Rev. Genet. 24: 363-385.

Belfort, M. and P.S. Perlman. 1995. Mechanisms of intron mobility. I. Biol. Chem. 270: 30237-30240.

Bell-Pedersen, D., S.M. Quirk, M. Aubrey, and M. Belfort. 1989. A site-specific endonuclease and co-conversion of flanking exons associated with the mobile $t d$ intron of phage $\mathrm{T} 4$. Gene 82: 119-126.

Bell-Pedersen, D., S. Quirk, J. Clyman, and M. Belfort. 1990. Intron mobility in phage $\mathrm{T} 4$ is dependent upon a distinctive class of endonucleases and independent of DNA sequences encoding the intron core: Mechanistic and evolutionary implications. Nucleic Acids Res. 18: 3763-3770.

Bell-Pedersen, D., S.M. Quirk, M. Bryk, and M. Belfort. 1991. I-TevI, the endonuclease encoded by the mobile $t d$ intron, recognizes binding and clcavage domains on its DNA target. Proc. Natl. Acad. Sci. 88: 7719-7723.

Bryk, M., S.M. Quirk, J.E. Mueller, N. Loizos, C. Lawrence, and M. Belfort. 1993. The $t d$ intron endonuclease makes extensive sequence tolerant contacts across the minor groove of its DNA target. EMBO /. 12: 2141-2149.

Bryk, M., M. Belisle, J.E. Mueller, and M. Belfort. 1995. Selection of a remote cleavage site by I-TevI, the $t d$ intron-encoded endonuclease. I. Mol. Biol. 247: 197-210.
Chu, F.K., G. Maley, J. Pedersen-Lane, A.-M. Wang, and F. Maley. 1990. Characterization of the restriction site of a prokaryotic intron-encoded endonuclease. Proc. Natl. Acad. Sci. 87: 3574-3578.

Clyman, J. and M. Belfort. 1992. Trans and cis requirements for intron mobility in a prokaryotic system. Genes \& Dev. 6: 1269-1279.

Dujon, B. 1989. Group I introns as mobile genetic elements: Facts and mechanistic speculations-a review. Gene 82: 91114.

George, J.W. and K.N. Kreuzer. 1996. Repair of double-strand breaks in bacteriophage $\mathrm{T} 4$ : Break-stimulated gene conversion in a coupled repair/replication reaction. Genetics 143: $1507-1520$.

Kleff, K. and B. Kemper. 1988. Initiation of heteroduplex-loop repair by $\mathrm{T} 4$-encoded endonuclease VII in vitro. EMBO $\%$. 7: 1527-1535.

Kreuzer, K.N. and B.M. Alberts. 1986. Characterization of a defective phage system for the analysis of bacteriophage T4 DNA replication origins. I. Mol. Biol. 188: 185-198.

Lambowitz, A.M. 1989. Infectious introns. Cell 56: 323-326.

Loizos, N., G.H. Silva, and M. Belfort. 1996. The intron-encoded endonuclcase I-TevII binds across the minor groove and induces two distinct conformational changes in its DNA substrate. I. Mol. Biol. 255: 412-424.

Morris, C.F., L.A. Moran, and B.M. Alberts. 1979. Purification of gene 41 protein of bacteriophage T4. 1. Biol. Chem. 254: 6797-6802.

Mueller, J.E., M. Bryk, N. Loizos, and M. Belfort. 1993. Homing endonucleases. In Nucleases (ed. S.M. Linn, R.S. Lloyd, and R.J. Roberts), pp. 111-143. Cold Spring Harbor Laboratory Press, Cold Spring Harbor, NY.

Mueller, J.E., D. Smith, M. Bryk, and M. Belfort. 1995. Intronencoded endonuclease I-TevI binds as a monomer to effect sequential cleavage via conformational changes in the $t d$ homing site. EMBO T. 14: 5724-5735.

Mueller, I.E., I. Clyman, Y. Huang, M.M. Parker, and M. Belfort. 1996. Intron mobility in phage $\mathrm{T} 4$ occurs in the context of recombination-dependent DNA replication by multiple pathways. Genes \& Dev. 10:351-364.

Parker, M.M., D.A. Court, K. Preiter, and M. Belfort. 1996. Homology requirements for double-strand break-mediated recombination in a phage lambda- $t d$ intron model system. $G e-$ netics 143: 1057-1068.

Perlman, P.S. and R.A. Butow. 1989. Mobile introns and intronencoded protcins. Science 246: 1106-1109.

Perrin, A., M. Buckle, and B. Dujon. 1993. Asymmetrical recognition and activity of the I-Scel endonuclease on its site and on intron-exon junctions. EMBO /. 12: 2939-2947.

Plessis, A., A. Perrin, J.E. Haber, and B. Dujon. 1992. Site-specific recombination detcrmined by I-Sce, a mitochondrial group I intron-encoded endonuclease expressed in the yeast nuclcus. Genetics 130: 451-460.

Sambrook, I., E.F. Fritsch, and T. Maniatis. 1989. Molecular cloning. A laboratory manual, Cold Spring Harbor Laboratory Press, Cold Spring Harbor, NY.

Selick, H.E., K.N. Kreuzer, and B.M. Alberts. 1988. The bacteriophage T4 insertion/substitution vector system. A method for introducing site-specific mutations into the virus chromosome. J. Biol. Chem. 263: 11336-11347.

Shcherbakov, V.P. and L.A. Plugina. 1991. Marker-dependent recombination in T4 bacteriophage. III. Structural prerequisites for marker discrimination. Genetics 128: 673-685.

Singer-Sam, I, and A.D. Riggs. 1993. Quantitative analysis of messenger RNA levels: Reverse transcription-polymerase chain reaction single nucleotide primer extension assay. 
Mueller et al.

Methods Enzymol. 225: 344-351.

Solaro, P.C., K. Birkenkamp, P. Pfeiffer, and B. Kemper. 1993. Endonuclease VII of phage T4 triggers mismatch correction in vitro. J. Mol. Biol. 230: 868-877.

Sweetser, D.B., H. Hough, J.F. Whelden, M. Arbuckle, and J.A. Nickoloff. 1994. Fine-resolution mapping of spontaneous and double-strand break-induced gene conversion tracts in Saccharomyces cerevisiae reveals reversible mitotic conversion polarity. Mol. Cell. Biol. 14: 3863-3875.

White, C.I. and J.E. Haber. 1990. Intermediates of recombination during mating type switching in Saccharomyces cerevisiae. EMBO I. 9: 663-673.

Wilson, G.G., K.K.Y. Young, and G.J. Edlin. 1979. High-frequency generalised transduction by bacteriophage T4. $\mathrm{Na}$ ture 280: $80-81$. 


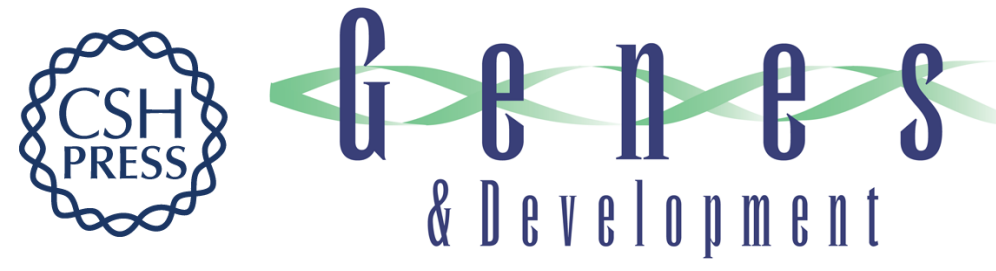

\section{Exon coconversion biases accompanying intron homing: battle of the nucleases.}

J E Mueller, D Smith and M Belfort

Genes Dev. 1996, 10:

Access the most recent version at doi:10.1101/gad.10.17.2158

References This article cites 29 articles, 13 of which can be accessed free at:

http://genesdev.cshlp.org/content/10/17/2158.full.html\#ref-list-1

License

Email Alerting

Service

Receive free email alerts when new articles cite this article - sign up in the box at the top right corner of the article or click here.

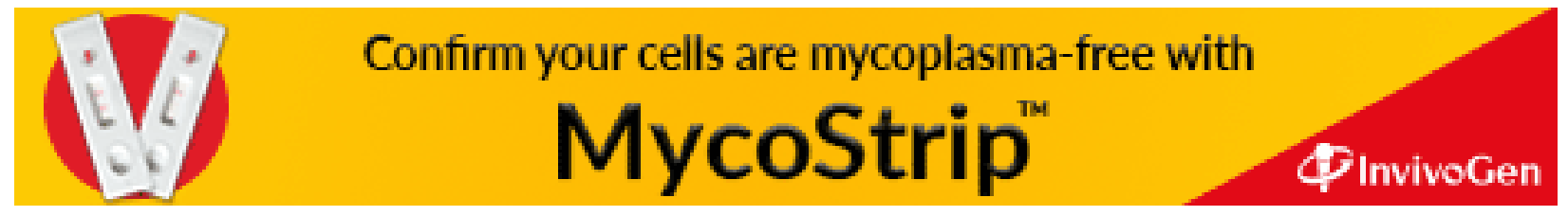

